Andrews University

Digital Commons @ Andrews University

Honors Theses

Undergraduate Research

$11-29-2018$

\title{
Assessing the Mean Neuronal Firing Rate Information Hypothesis via Mutual Information
}

Greg W. Zdor

greg@andrews.edu

Follow this and additional works at: https://digitalcommons.andrews.edu/honors

Part of the Computer Engineering Commons

\section{Recommended Citation}

Zdor, Greg W., "Assessing the Mean Neuronal Firing Rate Information Hypothesis via Mutual Information" (2018). Honors Theses. 217.

https://dx.doi.org/10.32597/honors/217

https://digitalcommons.andrews.edu/honors/217

This Honors Thesis is brought to you for free and open access by the Undergraduate Research at Digital Commons @ Andrews University. It has been accepted for inclusion in Honors Theses by an authorized administrator of Digital Commons@ Andrews University. For more information, please contact repository@andrews.edu. 
J.N. Andrews Honors Program

Andrews University

HONS 497

Honors Thesis

Assessing the Mean Neuronal Firing Rate Information Hypothesis via Mutual Information

Greg W. Zdor

Date: November $29^{\text {th }}, 2018$

Advisor: Jay R. Johnson

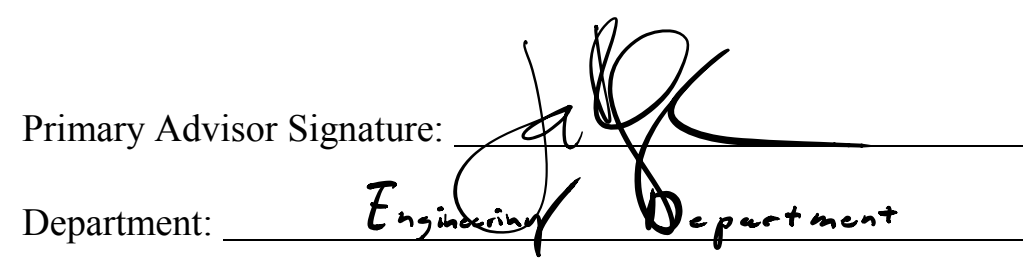




\title{
Assessing the Mean Neuronal Firing Rate Information Hypothesis via Mutual Information
}

\author{
Greg W. Zdor ${ }^{*}, 1$, Jay R. Johnson ${ }^{1}$, Ross K. Snider ${ }^{2}$ \\ Department of Engineering, Andrews University ${ }^{1}$ \\ Department of Electrical and Computer Engineering, Montana State University ${ }^{2}$
}

\begin{abstract}
While it is currently well accepted that the mean neuronal firing rate (MNFR) is a key parameter encoding information about sensory and motor events, in some cases the measured information due to MNFR is not adequate to explain the total neuron signal information content. In this study, several auditory neuron responses and corresponding MNFR—generated surrogates are analyzed using mutual information (MI) as a metric of information content. Results showed that for particular inter-spike gaps (ISG), data MI exceeded two standard deviations of the surrogate MNFR MI, indicating spike spacing and order also encode information.
\end{abstract}

*greg@andrews.edu 


\section{Introduction}

Neurons send information within the body of the neuron via electric signals called action potentials. These signals are in the form of slight variations in electric potential, or voltage, which are on the micro volt scale ${ }^{2}$. In 1926 researchers Adrian and Zotterman designed an electrical apparatus for recording the electrical activity in a nerve fiber. ${ }^{3}$ However, since the electrical signals from individual cells are very small, these signals needed amplification for analysis, which was provided in the early 1900 s by the valve vacuum tube amplifier. Now, with a way to amplify voltage signals, Adrian was able to analyze the signal output of neurons.

Adrian's experimentation laid the foundation for modern characterization of the neural response. While early experiments measured the response of a neuron by counting the number of spikes in a set period of time upon stimulus application to get a mean spike firing rate, today's experiments generally consist of measuring an ensemble of data measurements resulting from an identical stimulus and then performing ensemble averages. ${ }^{4}$

Figure 1. Shows the normalized voltage response of a marmoset auditory neuron after stimulus.

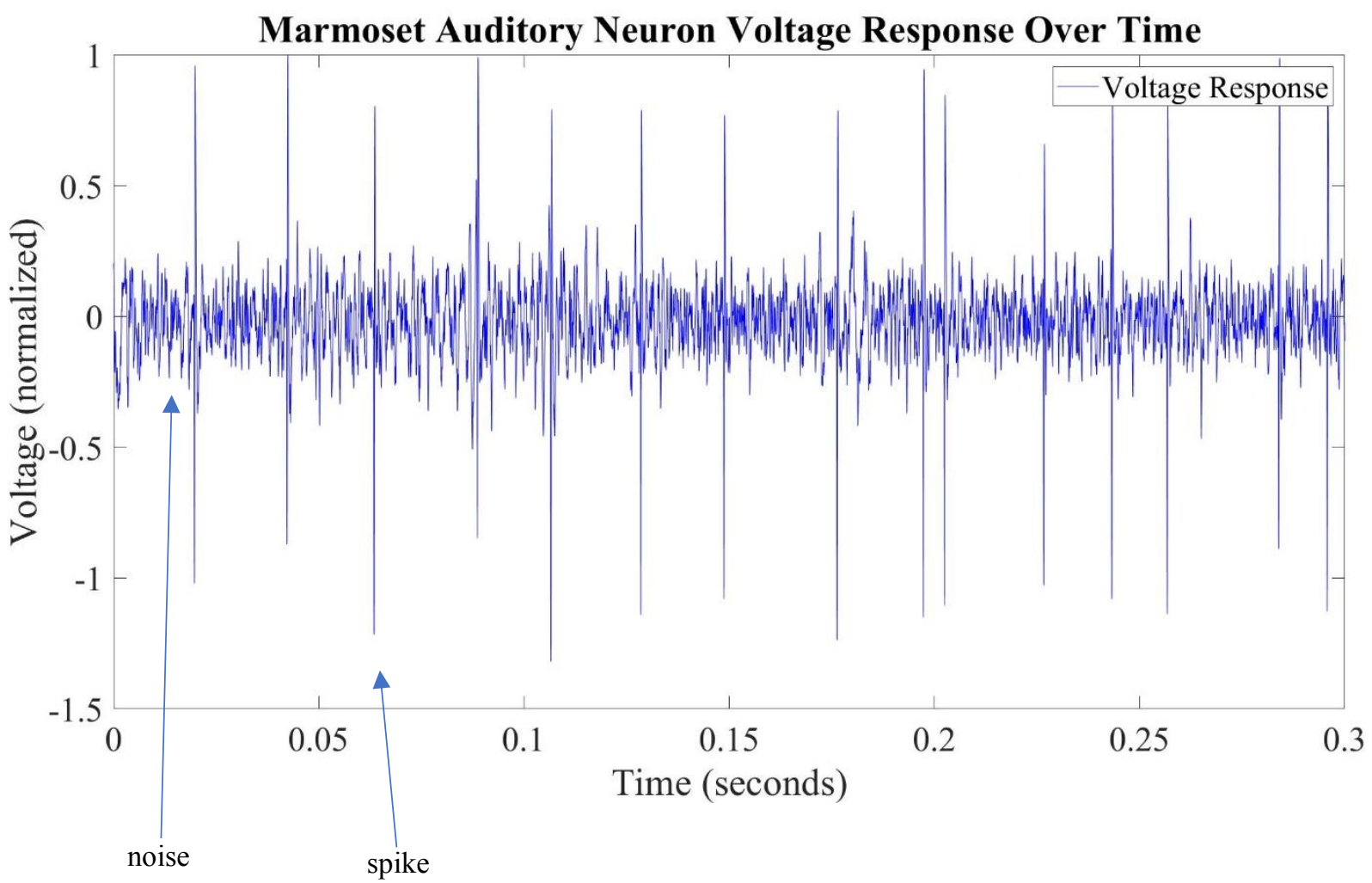

Considering Figure 1, if the signal was broken up into segments of equal lengths and then overlaid in plots, it would be apparent that the inter-spike gaps (ISG)s are not identical throughout trial. This variation in ISG widths indicates a presence of randomness in the neuron output signal. However, from Figure 1, it is evident that there is a minimum spike

\footnotetext{
${ }^{2}$ Rieke, F. Warland, D. Steeninck, R. Bialek, W. Spikes Exploring the Neural Code. MIT Press 1999 P. 5.

${ }^{3}$ Ibid. P. 10

${ }^{4}$ Ibid P. 19.
} 
spacing, a value that is in part determined by the brain's processing capacity. Therefore, directly after a voltage spike, the probability of there being a second spike decreases; however, as time passes the probability of a second spike increases in accordance with reaching the ISG width in time. Thus, while a given spike may deviate from the average ISG width, neural response on average is related to the average ISG width or its reciprocal, the mean neuronal firing rate (MNFR), as expressed below. ${ }^{5}$

$$
M N F R=\frac{\sum \text { spikes }}{\text { time interval }}
$$

Since Adrian and colleagues' discovery over ninety years ago, the fact that the MNFR encodes information about sensory and motor events is well-accepted fact. Recently, however, as Stein and colleagues point out, "others have questioned whether rate is the only parameter that encodes information about sensor and motor events." ${ }^{6}$ This query leads to the topic of my research.

The purpose of this study is to assess whether the MNFR encodes all the information content of a series of spike trains or if there is information that the MNFR does not account for. Therefore, the null hypothesis that all information content of the considered data set comes solely from the mean firing rate will be assessed using mutual information (MI) as the metric for information content. Data consisted of a single marmoset monkey's auditory neuron voltage outputs in response to an auditory stimulus. Surrogate data is generated from the MFR and its MI compared with the actual data's MI for a range of calculation parameters, allowing for assessment of the null hypothesis.

\section{Methodology}

\subsection{Data and Computation Tools}

The data set in the form of mat and .wav files was procured from Ross Snider at the Laboratory of Auditory Neurophysiology Department of Biomedical Engineering at John Hopkins University ${ }^{7}$. Data collection was funded via a National Science Foundation (NSF) grant, ensuring ethical recording procedures. Data collection was performed by attaching an electrode to an under-anesthetic marmoset monkey's auditory neuron and then playing an auditory recording of a marmoset monkey call to the monkey, which in turn stimulated the auditory neuron to fire. For each data collection, the same stimulus was applied multiple times, resulting in multiple neuron responses or realizations per file. Realization beginnings were denoted by the insertion of data markers with a magnitude 2 to 3 times the maximum neuron signal value, allowing for clear distinction between data marker and signal. Data consisted of 35 pairs of files, each pair consisting of the stimulus and the

\footnotetext{
${ }^{5}$ Borst, A. Juergen, H. "Effects of Mean Firing on Neural Information Rate." Journal of Computational Neuroscience. 10, $213-221,2001$.

${ }^{6}$ Stein, R. Gossen, R. Jones, K. "Neuronal Variability Noise or Part of the Signal?" Nature Reviews: Neuroscience. Vol 6. May 2005. P. $390-7$.

${ }^{7}$ Wanglab.johnshopkins.edu. (2018). Xiaoqin Wang, Ph.D. online. Available at: http://wanglab.johnshopkins.edu/HomePage.html Accessed 16 Nov. 2018.
} 
corresponding neural response to that particular stimulus. MATLAB, a numerical computing environment, was deployed as the tool for computation. See Appendix I for access to code used to perform this research.

Table 1. Summarizes the general recording parameters of the marmoset neuron response data.

\begin{tabular}{|l|l|}
\hline Sampling Rate (Fs) & 50,000 hertz \\
\hline Time Duration & 10 seconds \\
\hline Number of Realizations per File & Variable \\
\hline Number of Data Files & 35 \\
\hline
\end{tabular}

Figure 2. Shows the response signal versus time and spectrogram (Fast Fourier Transform (FFT) window size of 1024) plots for one of the data files analyzed. The red circles in the top figure indicate the new application (or a new realization) of the neuron stimulus, which in the case of this file there were 9 realizations.
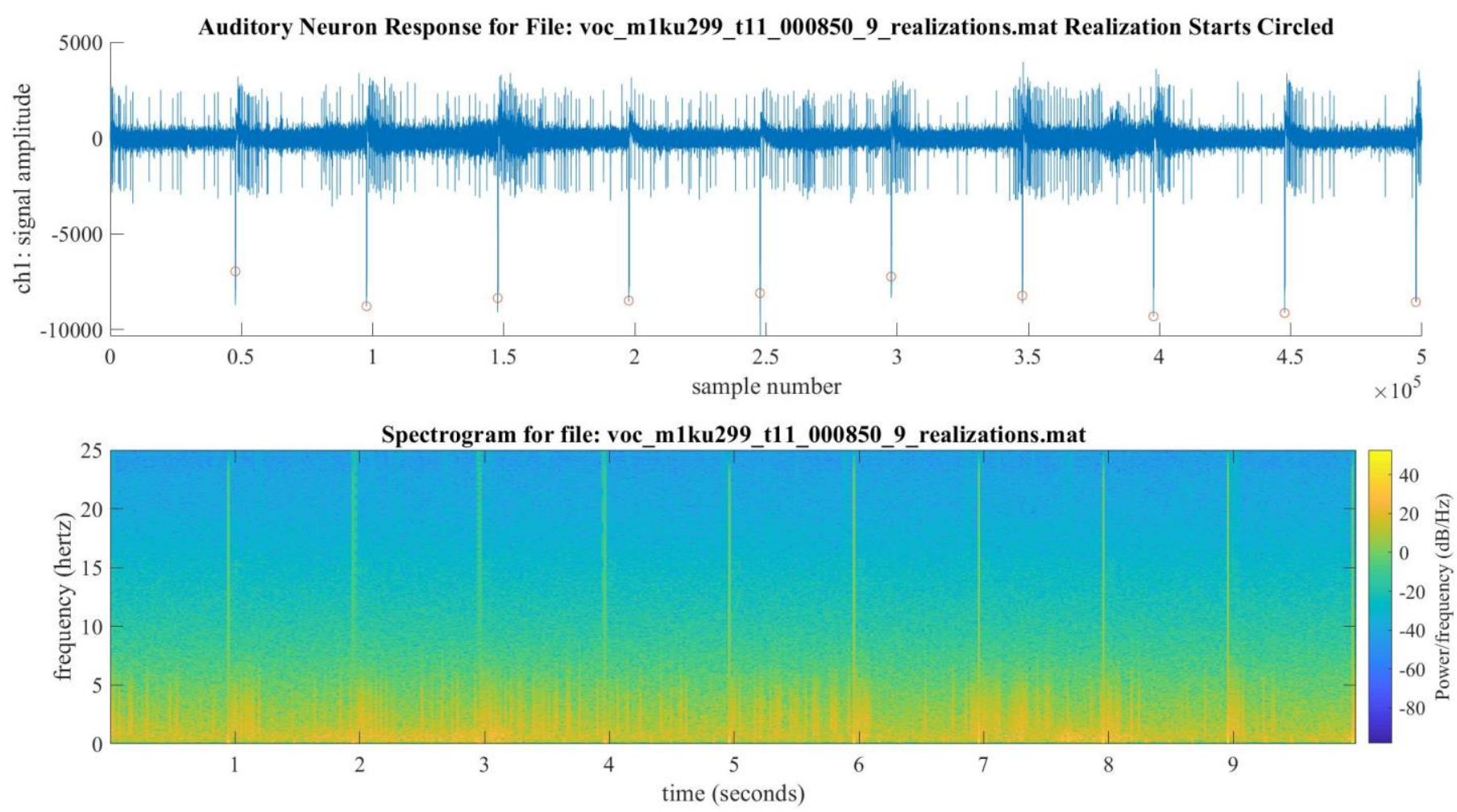
Figure 3. Shows the stimulus signal versus time and its corresponding spectrogram with at an FFT window size of 1024.
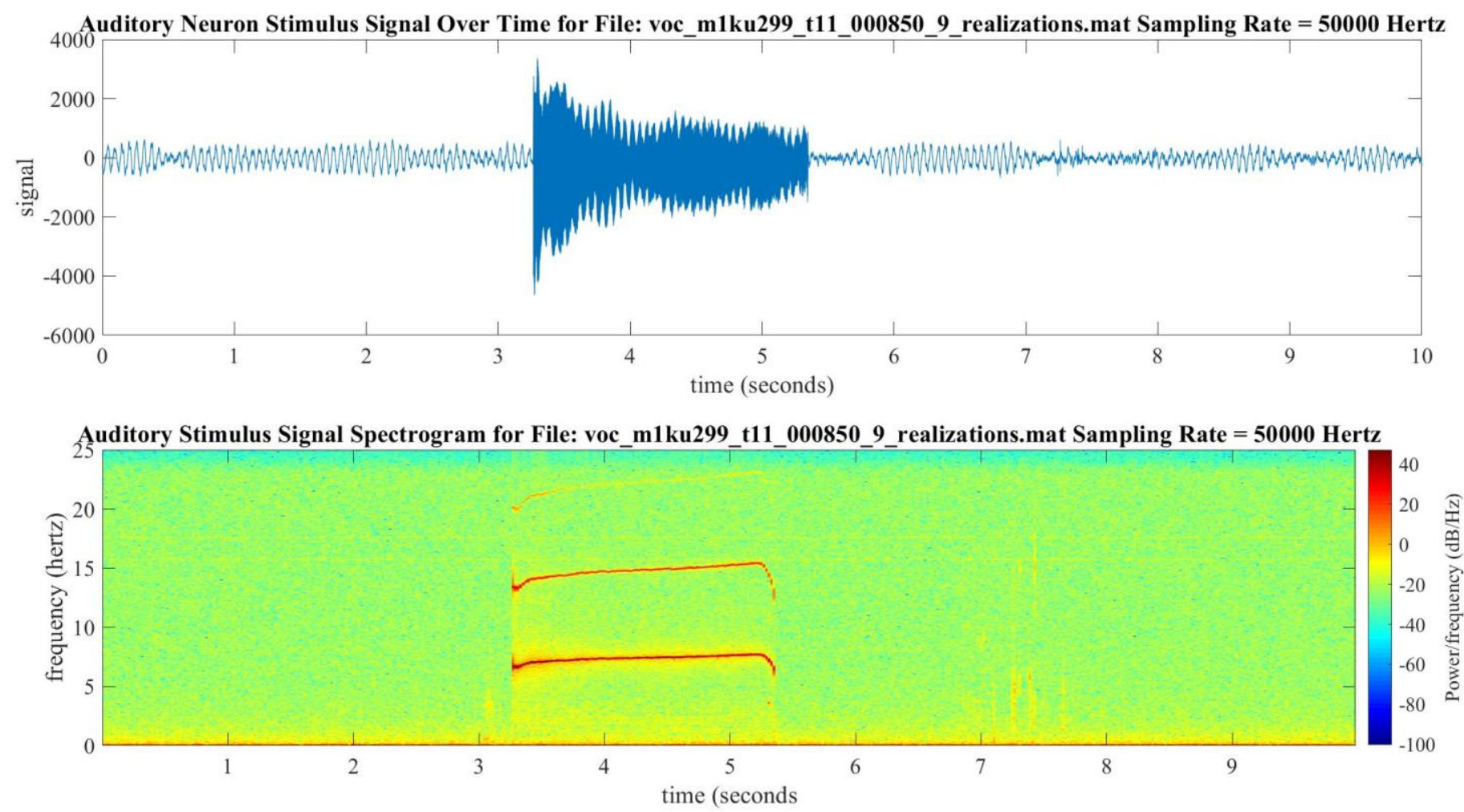

\subsection{Neuron Response Data Filtering}

In order to accurately calculate MI, realization beginning markers must be present in order to know where in time the stimulus application began. Therefore, presence of data makers was first checked for each file and files without realization markers were removed. Secondly, files were categorized by the number of realizations contained, where the definition of containing a realization demanded that a data marker be present at both beginning and end of the considered realization. Categorization results yielded 10 files with 3 realizations, 2 files with 9 realizations, and the remaining 23 files had less than 3 realizations. 
Figure 4. Shows a single realization where the beginning realization data marker amplitude $=\sim-8,000$ can be seen at sample number $=0$.

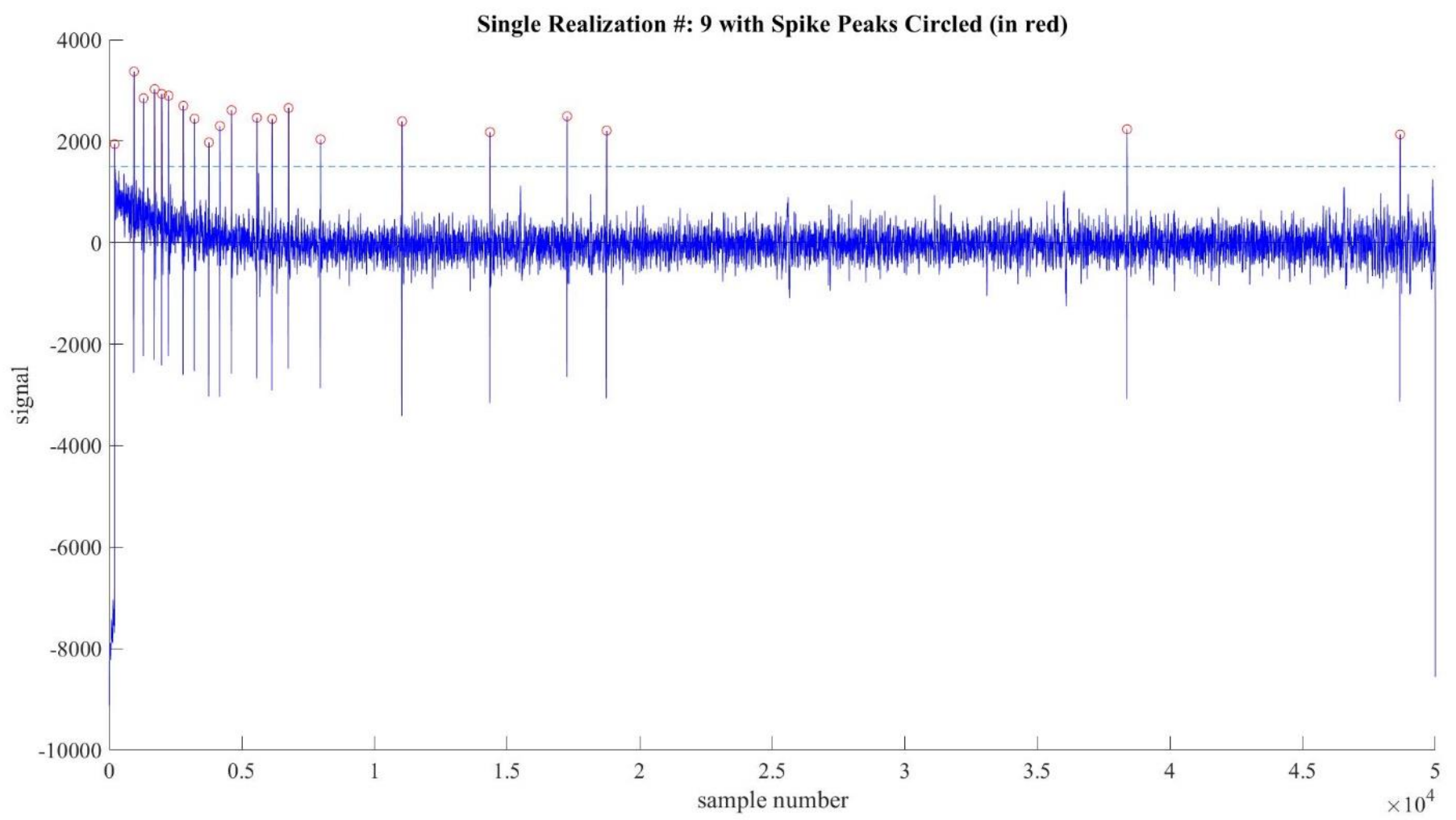

\subsection{Peak Detection}

A constant threshold-based detection method was deployed to identify spike peaks as Figure 3 above illustrates, where the dashed line denotes the detection threshold. Due to the high signal to noise ratio (SNR) in the data, the minimum height detection threshold was able to be set via observation to a value of 1500. Minimum peak separation (MPS) distance was another parameter used in spike detection. Because neurons have an estimated maximum firing rate (EMFR $)^{8}$ of 1.6 milliseconds, an MPS of 80 samples (sampling rate of 50K hertz) was used; equation (2) below shows MPS in sample number as a function of sampling rate and EMFR.

$$
M P S=(F S)(E M F R)
$$

Peak height and peak separation detection parameters were kept constant across all data files analyzed. Upon finding the peaks for each realization in a given data file, the ISG distance was calculated as the distance between adjoining peaks.

\footnotetext{
${ }^{8}$ Wang, B. et al. "Firing Frequency Maxima of Fast-Spiking Neurons in Human, Monkey, and Mouse Neocortex" Frontiers in Cellular Neuroscience. 2016; 10: 239. P.
} 


\subsection{Pulse Shape: Binary Encoding of Presence of Spike}

Figures 5 and 6. Show neuron signal pulse shape across all data files considered. The upper panel shows the average pulse shape for each of the considered data files, with the average pulse shape across all files plotted in the second subplot. The lower panel shows pulse shape invariant to pulse detection threshold. Consistent pulse shape allowed the presence of a pulse to be encoded binarily, where a 1 denoted presence of a spike, the maximal point of a pulse, and 0 represented non-spike data.

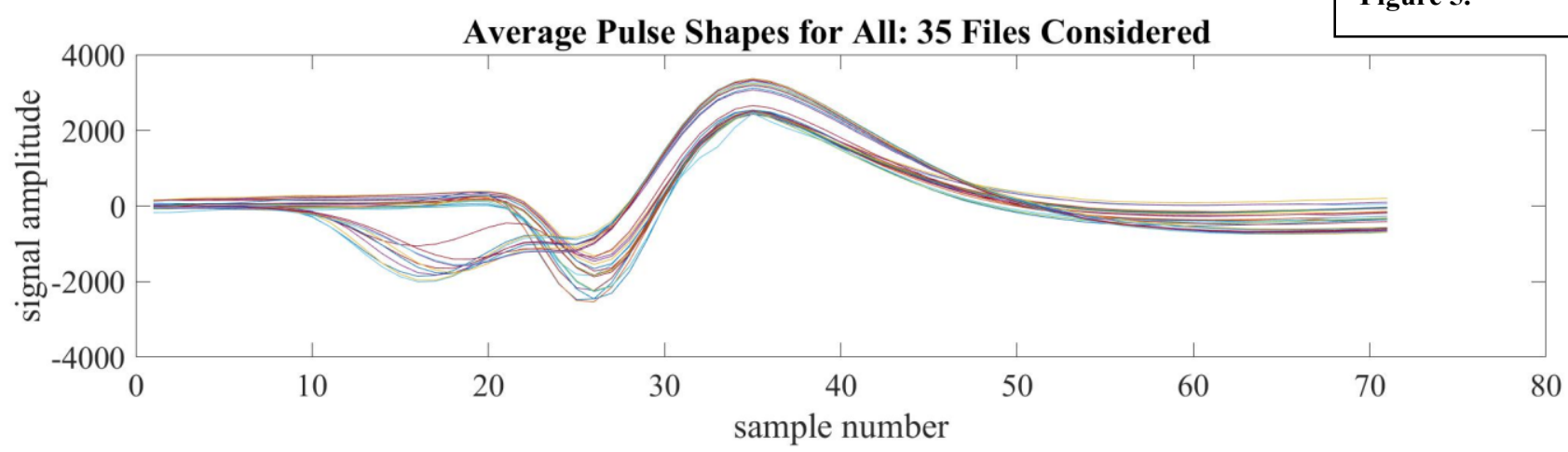

Average Spike Shape for All: 35 Files Considered: Pulse Threshold: 2100

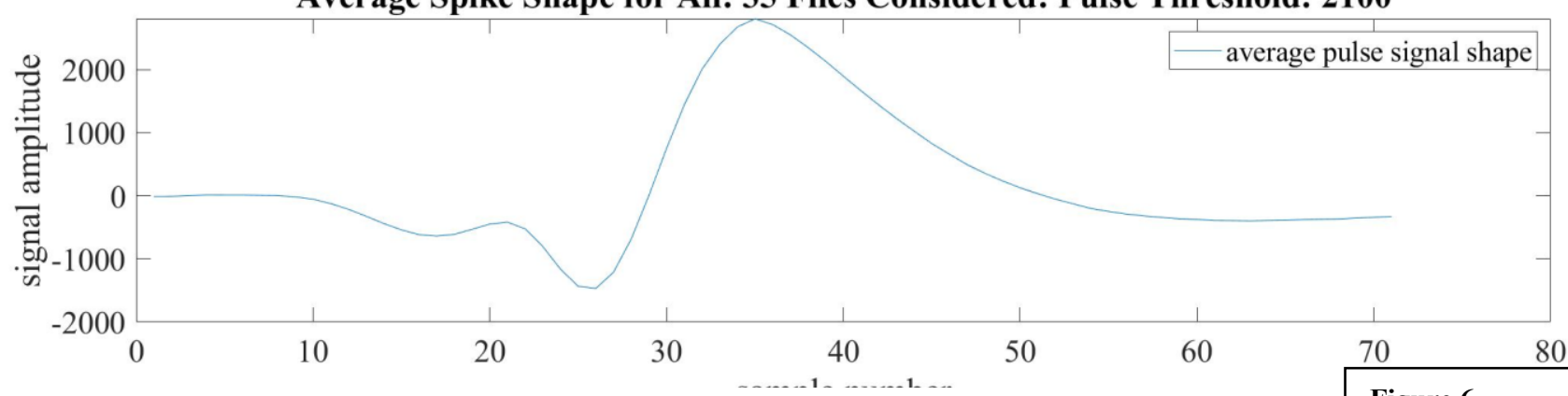

Figure 6.

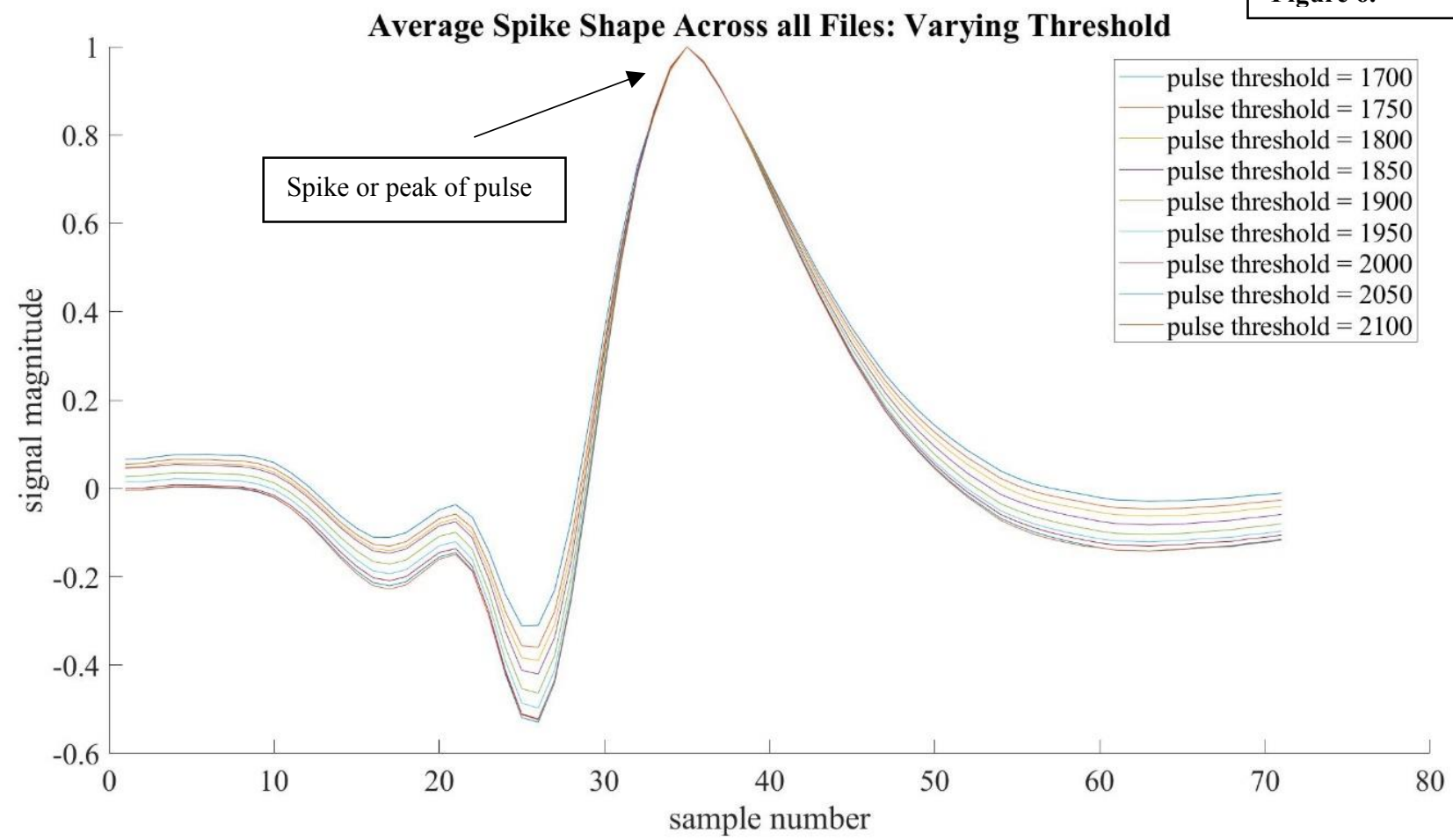




\subsection{Probability of Pulse Over Realization Indices}

For each data file, each realization data set was turned into a binary array, where a 1 was assigned to the presence of a pulse spike and a 0 to all other values. Aligning each realization's binary array over time and then summing contents over time generated a one-dimension array where each value in the array indicated the total number of spikes present across all realizations at that given realization index. Note, each realization binary array was truncated to the length of the shortest realization to ensure matching dimensions. Summing the values in this array gave the total number of spikes present in the given data file; consequent division of each array value by the total number of spikes present value yielded a probability density function (PDF) of having a spike in any given realization at a particular realization index, as Figure 7 illustrates in the lower plot. In order to better visualize the probability of pulse over sample number, the probability values were binned $^{9}$, resulting in the upper plot of Figure 7. Note that probability of spike does not monotonically decrease with bin number but rather initially decreases then has local maxima at bin numbers 15 and 38 .

Figure 7. Shows the probability of having a pulse at a particular instance in time during a realization versus realization index, where the upper plot shows the binned version of the lower plot's PDF, using a bin size of 1000 samples or 0.02 seconds.
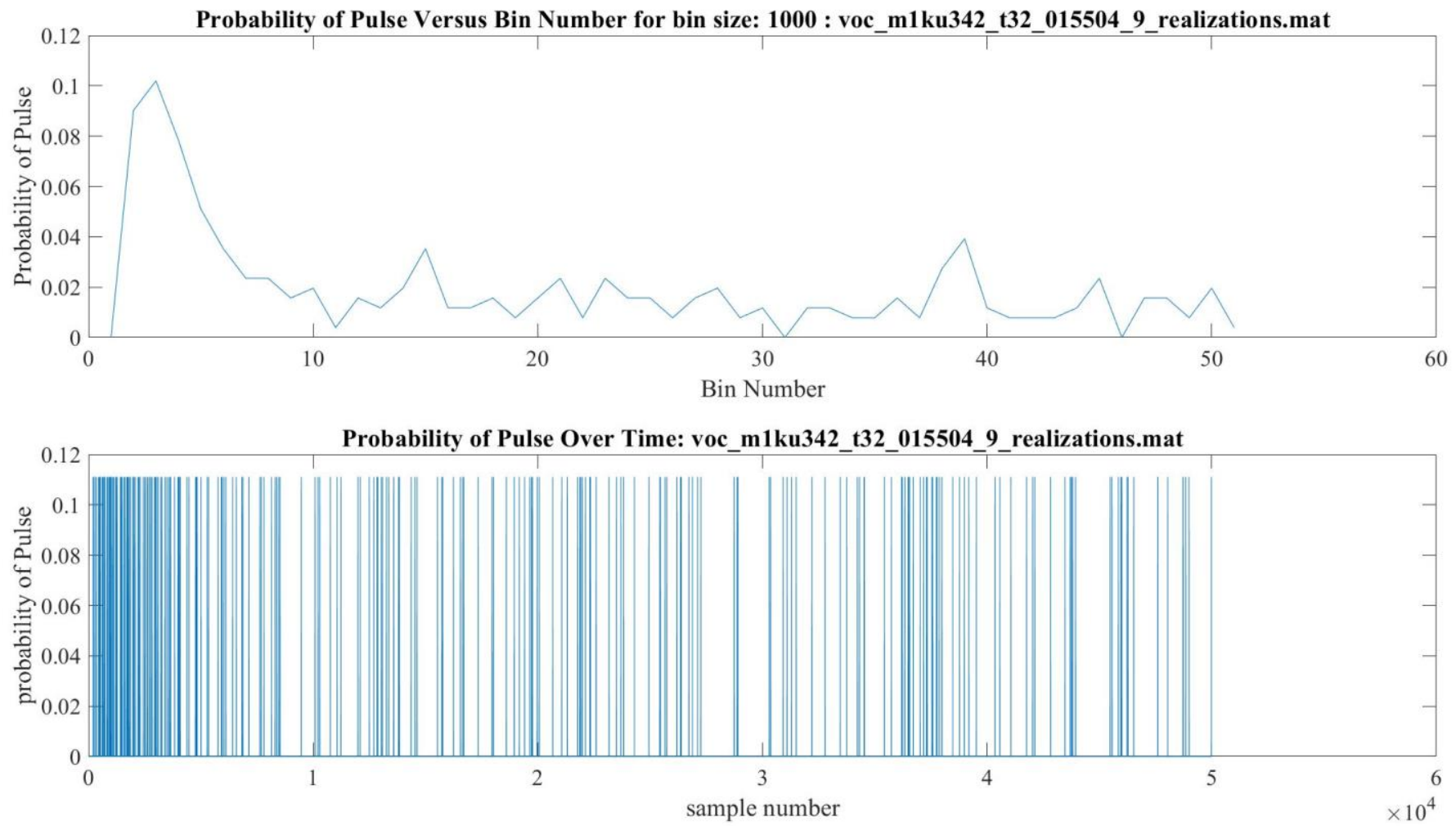

\footnotetext{
${ }^{9}$ See Section 2.7 for explanation on bin size choice
} 


\subsection{Realization Data Limitation}

From Figure 8 below, it may be seen that the resolution of the probability of a pulse was determined by the number of realizations per file. For example, for a file with 3 realizations the probability of a pulse over time could take on $1 / 3,2 / 3$ or $3 / 3$, while for a file with 9 realizations probability would increment in fractions of $1 / 9^{\text {th }}$. Therefore, the resolution for the probability of a pulse (RPP) was expressed as follows:

$R P P=\frac{1}{(\text { Number Realizations / file) }}$

With generating a probability density function that most accurately and precisely gave the probability of pulse as a function of realization sample number as the goal, maximizing RPP for the considered data set was done. Maximizing RPP meant only the 2 data files with 9 realizations were fit for analysis.

Additionally, since all data files were the same length in time, those files with 3 or less realizations inherently contained longer in time realizations than the files with 9 realizations. Comparing results between files with 3 realizations and files with 9 realizations therefore would not be possible.

Consequently, only the 2 data files with 9 realizations were analyzed in this study. The reduced size in data set was a limiting factor considered in the Conclusions sections of this paper.

Figure 8. Illustrates how the number of realizations per data file determined the RPP for the probability density function that gave probability of pulse as a function of realization length.

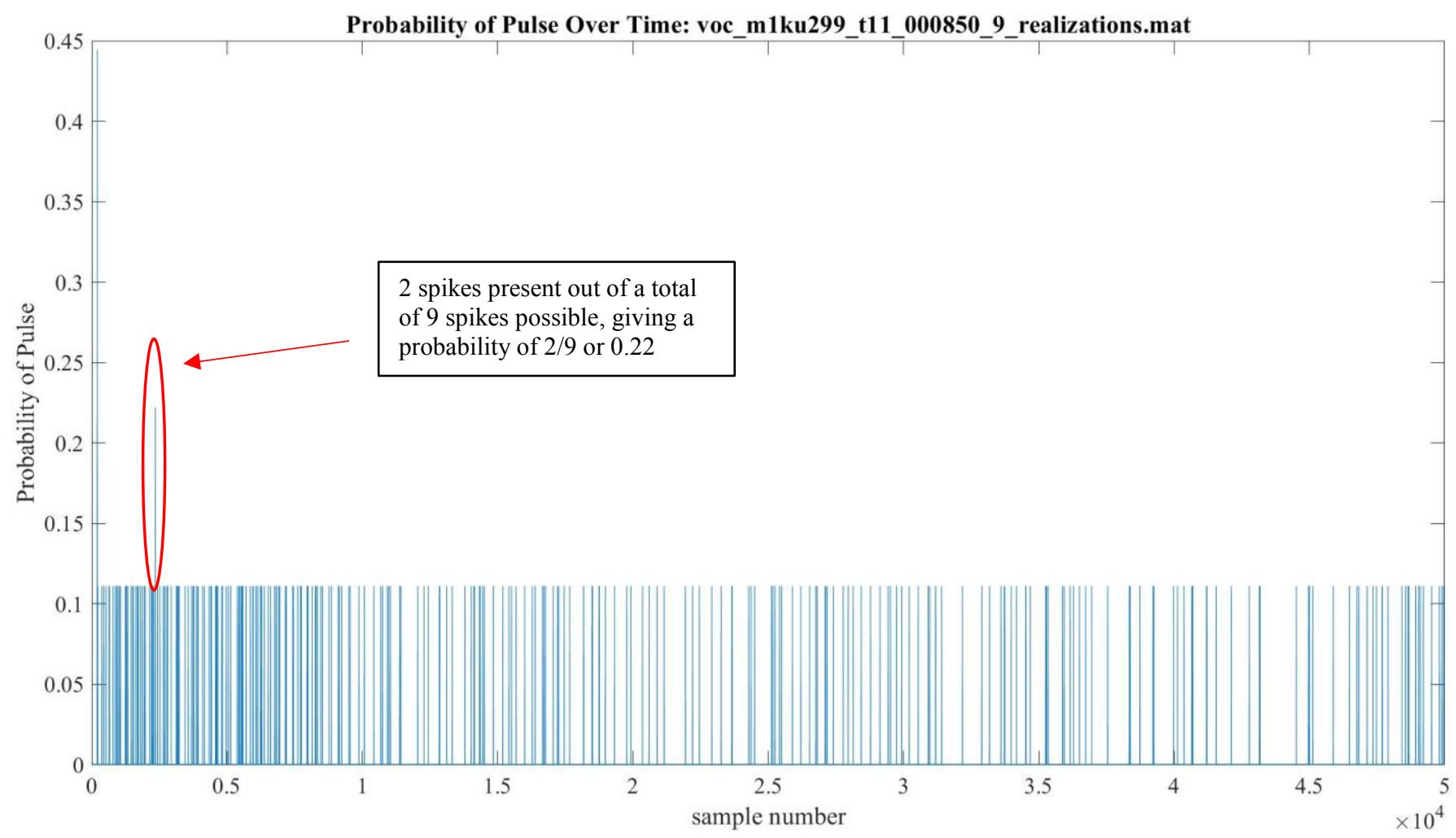




\subsection{Mutual Information Calculation: Non-Stationarity Approach}

\subsubsection{Across Look Ahead Calculation for Data}

Since Figure 7 clearly shows that the spike probability decreases over bin number, mutual information was calculated assuming non-stationarity of MNFR. Each realization was binned individually, a threshold set (must contain more than one spike) to define whether a bin contained a spike or not applied $(0=$ no spike; $1=$ contains spike $)$, and then the resulting binary matrix summed over bin number. Realization binning size was determined as follows:

- Constraints

○ Realization length: $\sim 50,000$ sample units

○ Minimum spike separation $=80$ sample units

○ Total number of bins $=($ realization length $) /($ minimum spike separation $)$

- Bin size range

- Minimum bin size: determined by minimum pulse separation value of 1.6 milliseconds or 80 sample numbers

○ Maximum bin size: determined by MNFR

- Explanation

- Bin size was varied starting at the minimum bin size and incrementally increased to upper bound for bin size: MNFR; MI results were compared as a function of bin size.

The binning yielded a time series $X$ for which MI was calculated using the following steps:

- Time Series Formatting and Binning

$\circ$ Define time series $X$ whose contents are the number of spikes per bin across all realizations

- Define a positive integer look ahead value (tau) for which to calculate MI in terms of

O Define a second time series $Y$ which is a time shifted - by - tau version of $X$

$\circ$ Define number of bins to use for binning as the maximum value in each time series

$\circ \quad$ Bin time series $X$ and $Y$

\section{- MI Calculations}

- Binning of time series $X$ and $Y$ resulted in each time series containing elements denoted by $\widehat{x(1)}, x \overline{(2)}, \ldots \widehat{x(M)}$ and $\widehat{y(1)}, \overline{y(2)}, \ldots \widehat{y(N)}$, respectively 
- Using a histogram binning method, the probability content of each bin $P_{b i n}(\widehat{x})$ and $P_{b i n}(\widehat{y})$ for dimensions $X$ and $Y$ respectively was calculated using:

$$
\begin{aligned}
& P_{b i n}\left(\widehat{x)}=\sum_{x(1)}^{x(M)} \frac{\hat{x}}{M}\right. \\
& P_{b i n}\left(\widehat{y)}=\sum_{y(1)}^{y(N)} \frac{\hat{y}}{N}\right.
\end{aligned}
$$

○ The total entropy $H(X)$ and $H(Y)$ respectively of each time series $X$ and $Y$ and the corresponding joint entropy $H(X, Y)$ was then calculated from the probability distribution function (PDF) of each bin using:

$$
\begin{aligned}
& H(X)=-\sum_{m=1}^{m=M} P_{(\text {bin } m)}\left(\widehat { x ) } \operatorname { l o g } \left(P_{(\text {bin } m)}(\widehat{x)})\right.\right. \\
& H(Y)=-\sum_{n=1}^{n=N} P_{(\text {bin } n)}\left(\widehat { y ) } \operatorname { l o g } \left(P_{(\text {bin } n)}(\widehat{y)})\right.\right. \\
& H(X, Y)=-\sum_{n=1, m=1}^{n=N, m=M} P_{(\text {bin } n, m)}\left(\widehat { ( x ) ( y ) ) } \operatorname { l o g } \left(P_{(\text {bin } n, m)}(\widehat{(x)(y))}\right.\right.
\end{aligned}
$$

We note also that the MI of the considered time series was dependent in part on the length of the time series. In turn, time series length is itself a variable of the tau used. Therefore, to account for MI changing as a function of time series length, the actual data and the surrogate time series lengths were changed equally with tau, allowing for comparison between actual and surrogate MI calculation results.

\subsubsection{Surrogate Data Generation and MI Calculation Over Tau}

Surrogate data (in the form of arrays replicating the number of actual data realizations and their length was created through randomly generating values between 0 and 1 and then using the MNFR to select the values at and below the MNFR value. Each realization was binned and then the same threshold as used for the actual data for defining a spike or not was applied to each bin, with spikes assigned 1 and non-spike bins assigned 0 . The resulting binary arrays were concatenated and summed over bin number, resulting in a time series for which MI was calculated in the exact same manner as the actual MI data calculation.

\subsubsection{Actual Data calculation for MI Over Time and Tau}

In order to examine the stationarity of the statistic, we also considered the variation of MI with time. This calculation was similar to how MI was calculated over tau solely; however, instead of binning each realization separately then applying a threshold to each bin to check for presence of a spike (as in 2.7.1), all realizations were summed over time and then binned, afterwards which a 
spike presence threshold was applied. MI was then calculated for the resulting time series with the time series beginning incremented from beginning to end bin.

\section{Results}

By deploying the steps outlined in section 2, a large number of plots were generated to determine the relationships between a variety of the parameters used and their corresponding outputs. The following pages in sections 3.1-3.2 illustrate key samples of some these results while section $\mathbf{3 . 3}$ provides a discussion of the results in the larger context of neural encoding.

\subsection{MI as a Function of Tau}

MI was calculated for both the actual data and the surrogate time series for a series of values of tau. Standard deviation of the surrogates at each tau value was then calculated, allowing for calculation of significance as a function of tau, where significance was defined as follows:

$\frac{M I(\text { data })-M I(\text { surr })}{S D(\text { surr })}$

Where SD denotes standard deviation and surr stands for surrogate.

Figure 9. Shows $\log _{2}$ of the number of occurrences for a given MI value for a given tau. This plot confirms that the randomly generated surrogate data fits a Gaussian distribution with large number of surrogates, in this case 10,000 surrogates.

MI Versus Tau and $\log (\mathrm{MI}$ ocurrences) for: voc_m1 ku342_t32_015504_9_realizations.mat Bin Size $=1000$ Number of Surrogates $=10000$

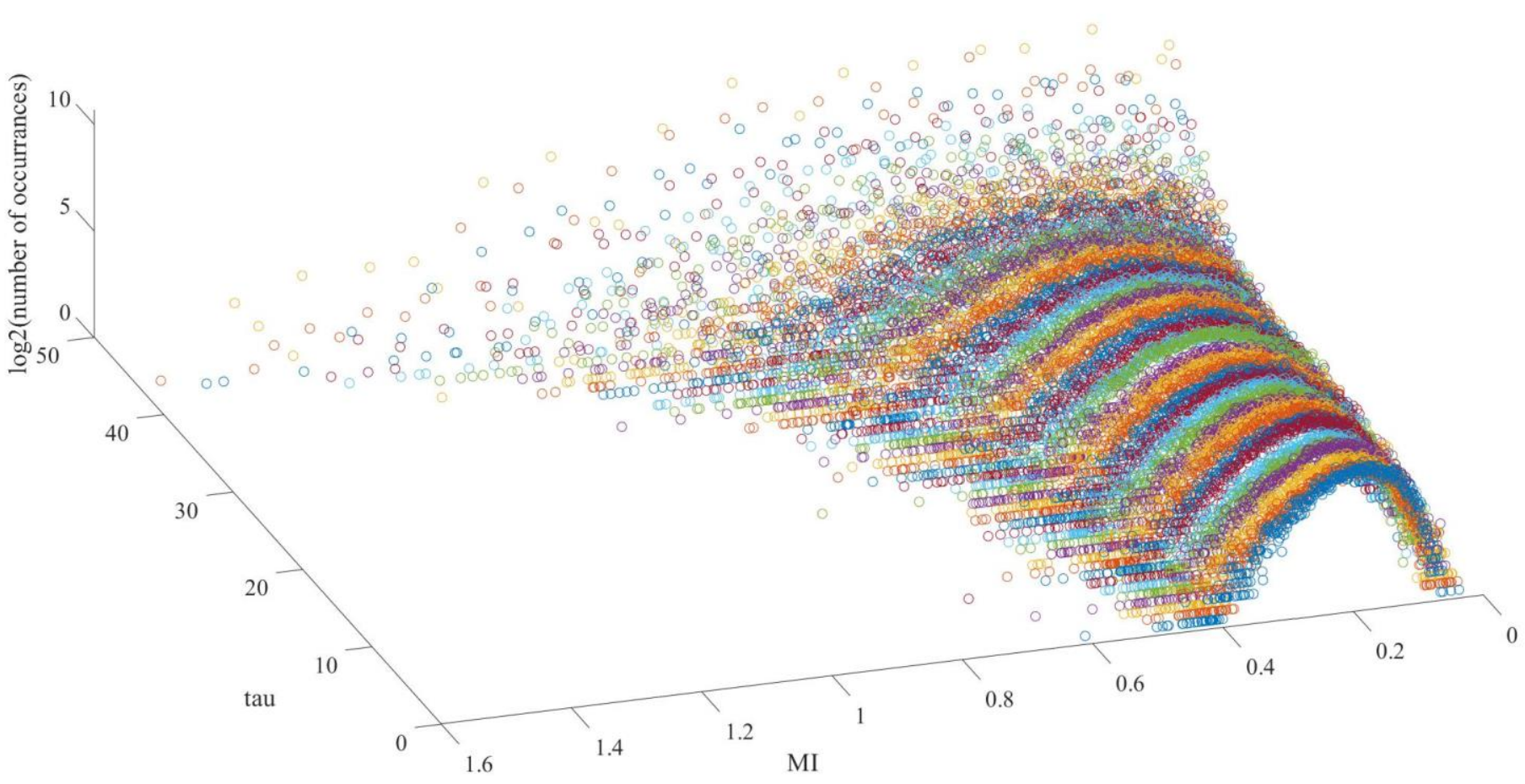


Figure 10. Shows the random distribution of the MI results for 10,000 surrogates, as well as illustrating the general trend that MI increased with increase in look ahead or tau. Figure 11. Contrasts the data MI versus the mean of surrogate data MI over tau for 10,000 surrogates. Note that for $(6<\operatorname{tau}<33)$ the actual data on average does not exceed one standard deviation of the surrogate data mean, but for tau equal to 38 and 41 the actual data exceeds the surrogate data by over two standard deviations, along with tau between 1 and 5 .

Surrogate MI Distribution for: voc_m1ku299_t11_000850_9_realizations.mat Bin Size = 1000

mutual information
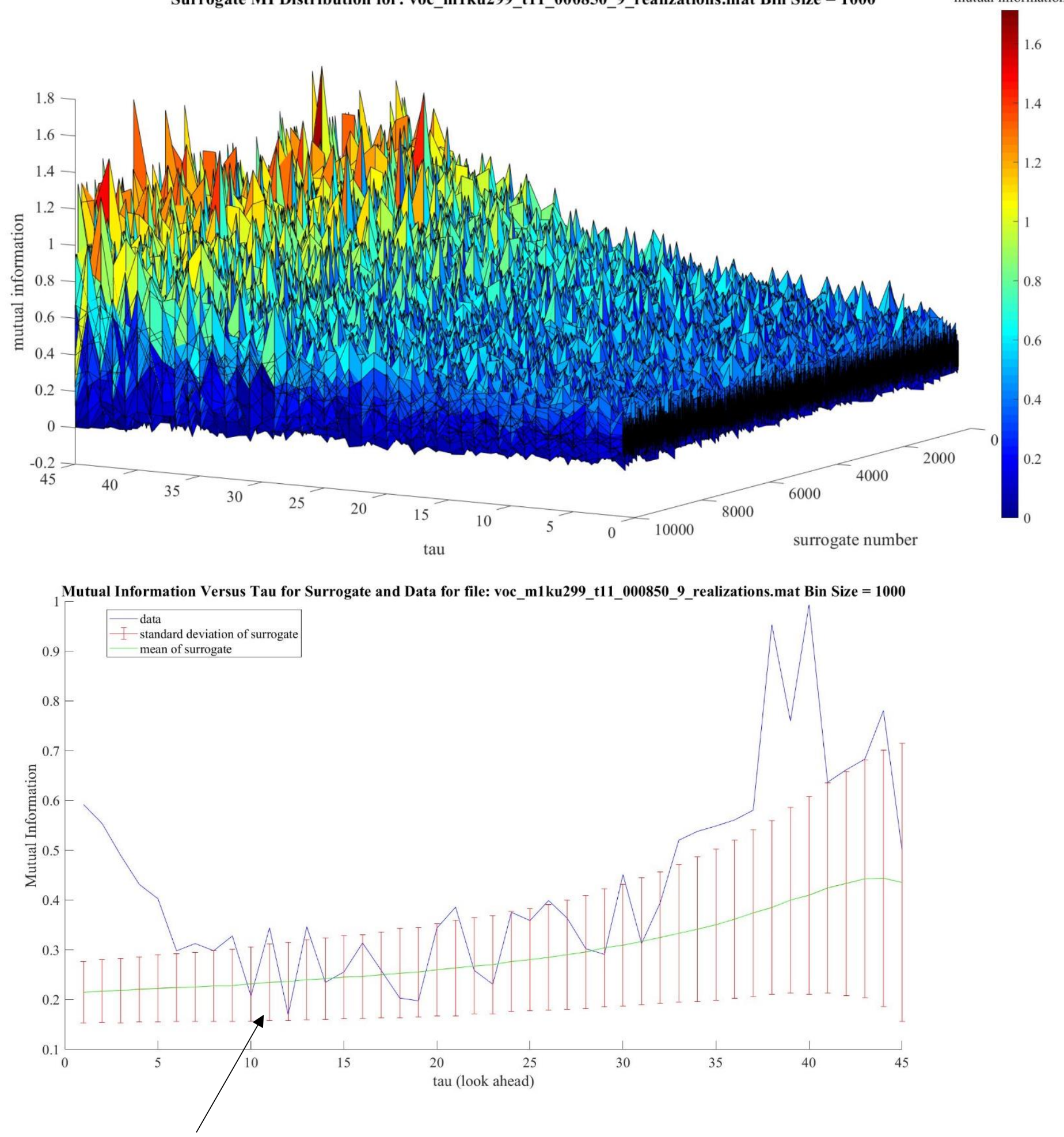

Mean of 10,000 surrogates (green) 
Figure 12. Shows the significance of the actual data MI values as a function of look ahead.

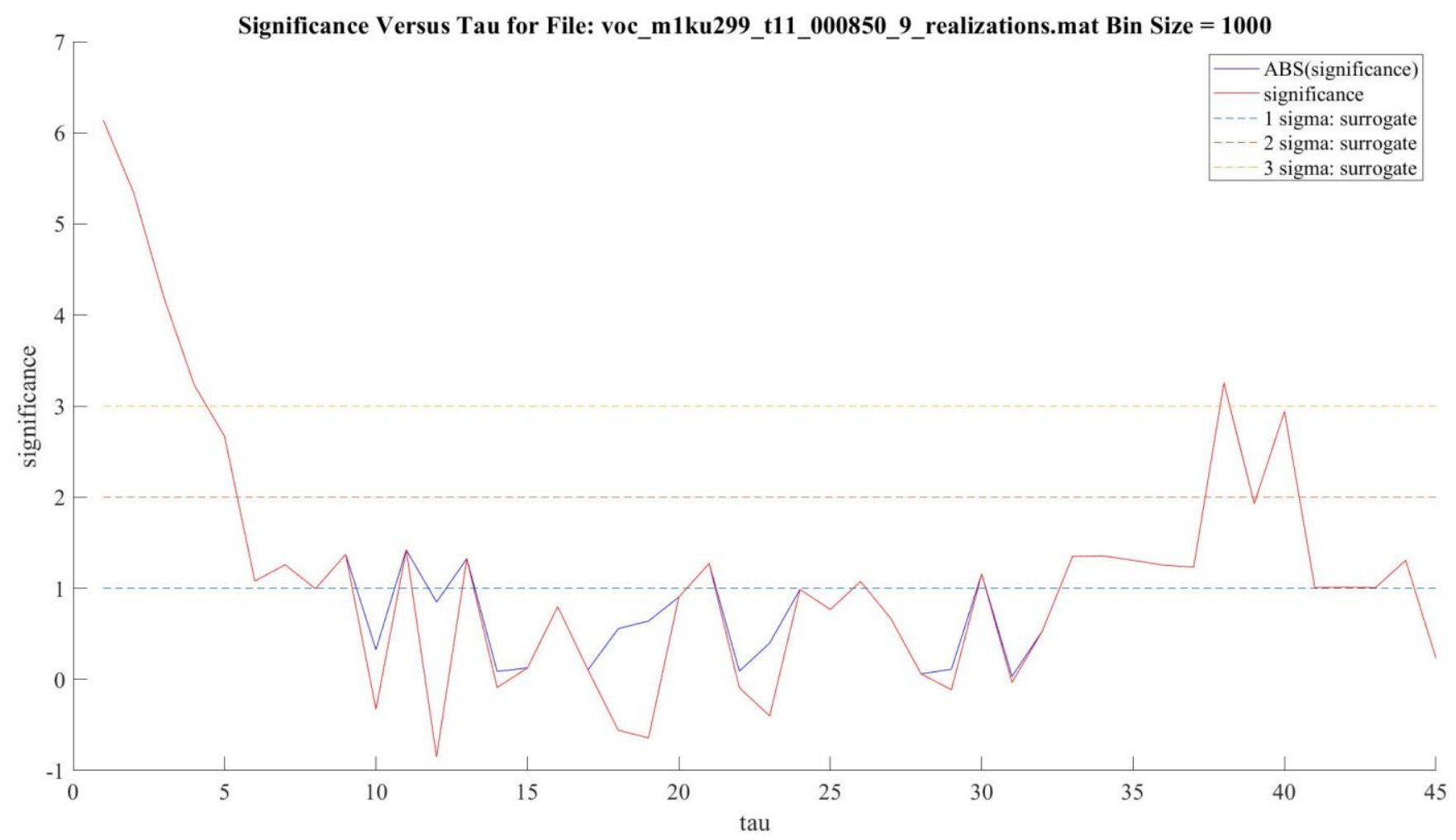

\subsection{Results: MI as a Function of Time and Tau}

Figure 13. Shows MI as a function of time at a bin size of 1000 .

MI Versus Tau and Time: voc_m1ku299_t11_000850_9_realizations.mat Bin Size $=1000$ Contains Spike Threshold $=5$

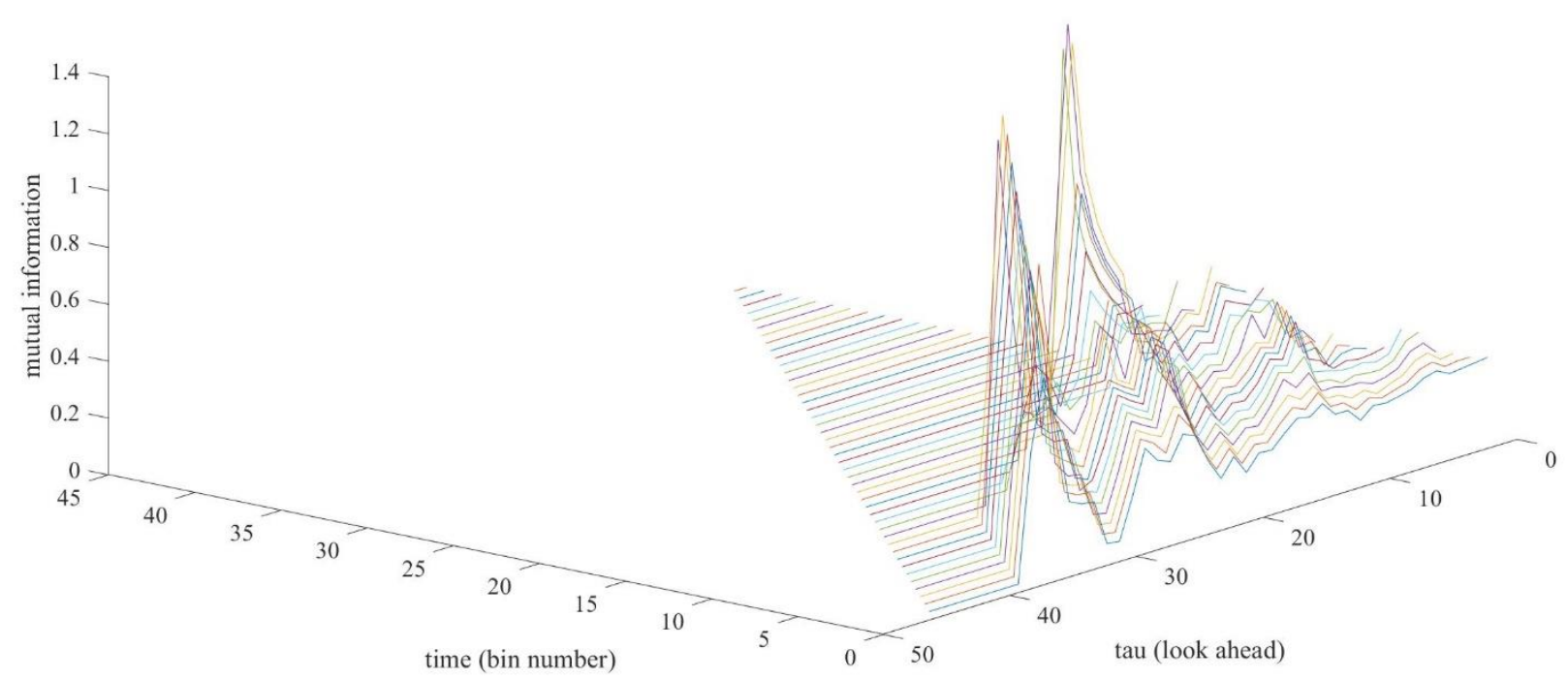


Figure 14. Shows the data MI versus tau time curves overlaid.

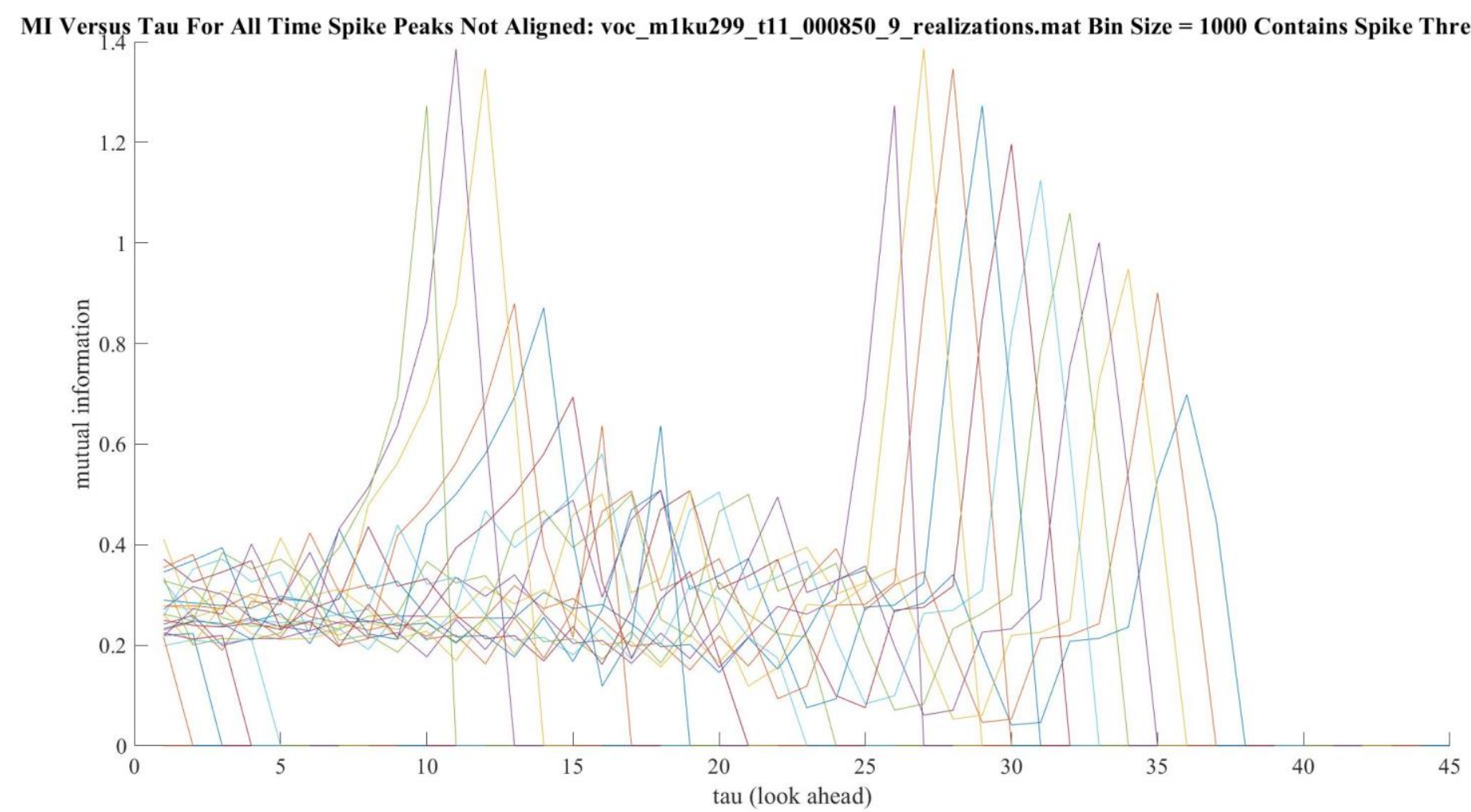

\subsection{Discussion and Interpretation of Results}

Results indicated that for particular look ahead values, the MNFR hypothesis-generated surrogate MI did not account for the MI of the data. Since MI was related to the bin size used, this input parameter was varied from a lower bound of 1.6 milliseconds or 80 samples to an upper bound of 33.6 milliseconds or 1680 samples. By varying bin size and seeing the significance of the MI did not change over bin size but rather only as a function of time separation ${ }^{10}$, it was determined that significance was invariant to bin size. ${ }^{11} \mathrm{MI}$ was not invariant to time series length however, indicated by the change in the standard deviation envelope of the surrogate in Figure 11 where it increases as the time series shortens. This particular observation leads to a larger problem encountered in this research: a shortage of data. Of the 35 files available, only 2 files or $5.7 \%$ of the data was usable due to containing limited number of realizations. Moreover, the short realization lengths in the usable data meant that varying tau also meant varying time series length by up to $50 \%$, while ideally time series length should be kept constant. We did to try control for this varying time series length by ensuring both data and surrogate time series lengths changes proportionally. MI as a function time in Figure 13 and 14 indicated that the data MI did not vary as a function of where in the time series $t=0$ or the beginning of the time series was defined, indicating MI as appearing invariant to time. However, we did not explore the relationship of MI and time in more depth as the primary focus of this research was assessing MI as a function of tau.

\footnotetext{
${ }^{10}$ Bin size multiplied by tau gives a value in time. Therefore, so long as this product is constant and where the data MI spikes above the surrogate MI is at a constant time even if bin size varies, then MI is invariant to bin size.

${ }^{11}$ Varying bin size was performed to verify lack of dependence on significance but these plots are not shown.
} 
Currently, there is much debate in the field of neural encoding in neuroscience between proposed coding schemes for a train or sequence of neuron signal spikes. ${ }^{12}$ The MNFR hypothesis being assessed in this study fits within the coding scheme of rate encoding. In contrast, a temporal encoding scheme is one based on the specific timing of single spikes, sometimes in direct connection to an outside stimulus. ${ }^{13}$ While the results of this study need more verification by analyzing more data, preliminary results indicate that in the case of a single Marmoset auditory neuron, rate encoding does not account for the considered spike train's information content. As Figure 7 in the lower plots indicates, there are repeated periods of higher spike probability at bins 15 and 38; from a rate encoding view these points should be counted as noise; however, temporal encoding might suggest their precise location in time encodes information as well. We conclude that further testing of the MNFR should be performed on a larger data set before decisive conclusions are drawn from this research.

\section{Conclusion and Future Work}

Results showed spikes had a significant impact on predicting subsequent spikes for large timescales of (0.6 to 0.8 seconds) and short time scales (0.02 to 0.1 seconds). Subsequently, we have shown that the MNFR hypothesis does not account for all information in the considered data set. However, since the considered data set was only two data files, future work will include expanding the data set and increasing number of and length of realizations per data recording. Other future work includes exploring the relationship between MI and optimal bin size and assessing MI at a farther out future time (larger look ahead). Since the MATLAB analysis scripts for this project were written in a manner to handle variable number of input files, this research has also resulted in the development of a scalable tool others may deploy in neuron signal analysis.

\section{Acknowledgements}

Special thanks go to Dr. Jay R. Johnson as primary advisor on this project and Dr. Ross K. Snider as collaborator and for providing the data set. Additionally, Dr. Shandelle M. Henson of the Andrews University Mathematics Department provided insight on surrogate data generation. Moreover, Dr. Giuseppe Passucci of SRC Inc. indirectly provided MATLAB instruction. Indirect funding included the Office of Research and Creative Scholarship at Andrews University and Dr. Johnson's NASA Grant \#: NNX 16AQ87G.

\footnotetext{
${ }^{12}$ Ventura, V. Carta, R. “Statistical Analysis of Temporal Evolution in Single-Neuron Firing Rates.” Biostatistics. $2002 ; 3,1, \mathrm{p} 1$.

${ }^{13}$ Ibid. 2. 


\section{Appendix I}

See following links to access PDF files of the following scripts and functions developed as part of this research:

- Function for generating average pulse shape across all files

○ https://drive.google.com/open?id=1Gula13nzxldK3N5dwK4Ig1HTBPaGqLf8

- Script for testing average pulse function for different pulse detection thresholds

○ https://drive.google.com/open?id=10x4tlxma5iZEgXKoYX1lnbkSzBp2q7g0

- Script "master" or main data pipeline through which to perform all calculations

○ https://drive.google.com/open?id=1q721kvJYN4DGEDZzll-ixcwn7U1SDeDB

- Function for mutual information calculation

○ https://drive.google.com/open?id=10fnDotnXiRDAtyNQzMuOcKcw_slBx-be

- Function for Shannon entropy calculation

○ https://drive.google.com/open?id=1P8QUQ6zy3iIv4AYJUiuiPQIBDXB4D9gc

\section{References}

1. Borst, A. Juergen, H. “Effects of Mean Firing on Neural Information Rate.” Journal of Computational Neuroscience. 10, 213-221, 2001.

2. Rieke, F. Warland, D. Steeninck, R. Bialek, W. Spikes Exploring the Neural Code. MIT Press 1999.

3. Stein, R. Gossen, R. Jones, K. "Neuronal Variability Noise or Part of the Signal?" Nature Reviews: Neuroscience. Vol 6. May 2005. P. 390-7.

4. Wang, B. et al. "Firing Frequency Maxima of Fast-Spiking Neurons in Human, Monkey, and Mouse Neocortex" Frontiers in Cellular Neuroscience. 2016; 10: 239.

5. Wanglab.johnshopkins.edu. (2018). Xiaoqin Wang, Ph.D. online. Available at: http://wanglab.johnshopkins.edu/HomePage.html Accessed 16 Nov. 2018.

6. Ventura, V. Carta, R. "Statistical Analysis of Temporal Evolution in Single-Neuron Firing Rates.” Biostatistics. $2002 ; 3,1, \mathrm{p} 1$. 